\title{
Estudio de cromóforos orgánicos con propiedades ópticas no lineales
}

\author{
P. ACEBAL, S. BLAYA, L. CARRETERO \\ Dept. de Ciencia y Tecnología de Materiales (Div. Óptica). Universidad Miguel Hernández
}

\begin{abstract}
Second-order nonlinear optical properties of three dyes (methylene blue, thionine and coumarin 7) were evaluated using $a b$ initio quantum mechanical methods and compared with those of a standard push-pull chromophores (disperse red 1, DMNPAA and PNA). The two level model was used to obtain the frequency dependency of the microscopic electrooptic effect, and the results show that the obtained values for the dyes are higher than the standard chromophores at wavelengths of the near infrared. Therefore, these dyes may be applied in photonic devices and photorefractive materials.
\end{abstract}

Palabras clave: materiales fotorrefractivos, óptica no lineal, ab initio, colorantes.

\section{Study of organic chromophores with non linear optical properties.}

Mediante métodos de cálculo ab initio se han estudiado las propiedades ópticas no lineales de segundo orden de tres colorantes (azul de metileno, tionina y coumarina 7), y se han comparado con los obtenidos para tres cromóforos donador-aceptor (disperse red 1, DMNPAA y PNA). Posteriormente se ha utilizado el modelo de los dos niveles para estimar la dependencia con la frecuencia del efecto electroóptico microscópico. Los resultados muestran que los colorantes estudiados tienen valores de esta propiedad del mismo orden o superiores que los cromóforos clásicos en longitudes de onda del infrarrojo cercano, por lo tanto puede estudiarse su aplicación en dispositivos fotónicos y materiales fotorrefractivos.

Keywords: fotorefractive materials, non linear optics, ab initio, dyes.

\section{INTRODUCCIÓN}

El desarrollo de nuevos materiales con propiedades ópticas no lineales (NLO) ha sido uno de los principales objetivos de la investigación durante las últimas décadas, debido a sus importantes aplicaciones en fotónica [1] o en el almacenamiento óptico de información [2,3]. Dentro de este tipo de materiales, en los últimos años ha surgido un gran interés en los compuestos orgánicos, puesto que poseen ventajas frente a los cristales inorgánicos, como son su bajo coste, facilidad de procesado y alto número de combinaciones, que los hacen muy atractivos para la industria.

Generalmente los materiales orgánicos están compuestos por una matriz polimérica en la que se distribuyen los cromóforos que aportan las propiedades ópticas no lineales, encontrándose en ocasiones estas moléculas directamente enlazadas al polímero [4]. Las propiedades ópticas macroscópicas del material son, por tanto, función de las correspondientes microscópicas de los cromóforos empleados [5], por lo que una de las principales formas de optimización del material consiste en la búsqueda de moléculas con altos valores de sus propiedades microscópicas [6,7]. En este sentido se han desarrollado numerosos trabajos, siendo la principal estrategia el empleo de los compuestos denominados push-pull, que están formados por una cadena de dobles enlaces conjugados con un grupo donador de electrones en un extremo y un grupo aceptor en el otro. A este grupo pertenecen compuestos como la $p$-nitroanilina (PNA), el 2-[etil[4-[(4-nitrofenil)azo]fenil]amino ]etanol (DR1) y el 2,5-dimetil-4-(p-fenilazo)anisol (DMNPAA), que se pueden observar en la figura 1 . Dentro de éstos, la PNA es la molécula de referencia en cuanto a las propiedades NLO, puesto que constituye uno de los sistemas push-pull más simples, y es uno de los más estudiados. En alternativa a este tipo de compuestos en este trabajo hemos estudiado las propiedades NLO de moléculas clásicamente empleadas como colorantes, en concreto dos de la familia de las fenotiazinas, el azul de metileno (BM) y la tionina (TIO), y la coumarina 7 (COU7)

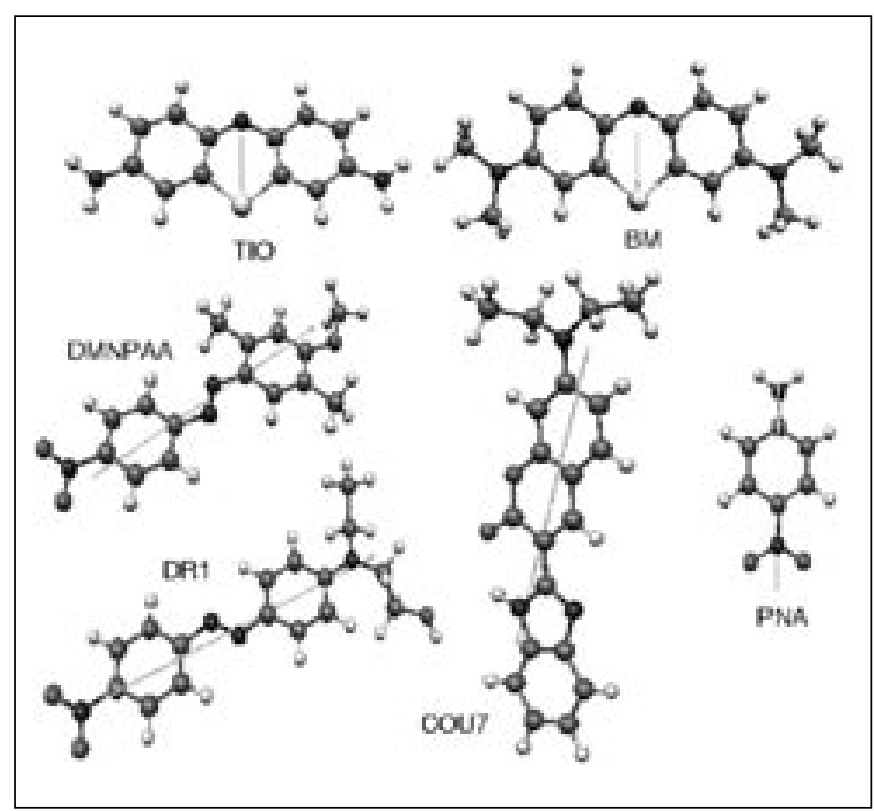

Figura 1: Moléculas estudiadas en este trabajo (Verde $=\mathrm{C}, \mathrm{Azul}=\mathrm{N}$, Rojo $=\mathrm{O}$, Amarillo=S, Blanco=H). La flecha naranja simboliza la dirección de momento dipolar molecular (eje z).

(figura 1). El estudio de las propiedades NLO de las moléculas seleccionadas se ha realizado en este trabajo mediante cálculos de mecánica cuántica, que son una buena alternativa a los métodos experimentales para el estudio y diseño de nuevas moléculas con propiedades ópticas no lineales $[8,9,10,11]$. 


\section{DESCRIPCIÓN TEÓRICA}

Las propiedades ópticas microscópicas se definen mediante sendos desarrollos en serie de Taylor de la energía y del momento dipolar molecular respecto al campo eléctrico aplicado $(\mathrm{F})$ :

$\mu_{\mathrm{i}}=\mu_{\mathrm{g}, \mathrm{i}}+\varepsilon_{\mathrm{o}}\left(\alpha_{\mathrm{ij}}(\omega ; \omega) \mathrm{F}_{\mathrm{j}}(\omega)+1 / 2 \beta_{\mathrm{ijk}}\left(-\omega ; \omega_{1^{\prime}}, \omega_{2}\right) \mathrm{F}_{\mathrm{j}}(\omega) \mathrm{F}_{\mathrm{k}}(\omega)+1 / 6 \chi_{\mathrm{ijk}}\left(-\omega ; \omega_{1}\right.\right.$ $\left.\left.\omega_{2^{\prime}} \omega_{3}\right) \mathrm{F}_{\mathrm{j}}(\omega) \mathrm{F}_{\mathrm{k}}(\omega) \mathrm{F}_{1}(\omega)+\ldots\right)$

$\mathrm{E}=\mathrm{E}_{\mathrm{o}}+\mu_{\mathrm{g}, \mathrm{i}} \mathrm{F}_{\mathrm{i}}(\omega)+\varepsilon_{\mathrm{o}}\left(\alpha_{\mathrm{ij}}(\omega ; \omega) \quad \mathrm{F}_{\mathrm{i}}(\omega) \mathrm{F}_{\mathrm{j}}(\omega)+1 / 2 \quad \beta_{\mathrm{ijk}}\left(-\omega ; \omega_{1}, \quad \omega_{2}\right)\right.$ $\left.\mathrm{F}_{\mathrm{i}}(\omega) \mathrm{F}_{\mathrm{j}}(\omega) \mathrm{F}_{\mathrm{k}}(\omega)+1 / 6 \chi_{\mathrm{ijkl}}\left(-\omega ; \omega_{1}, \omega_{2^{\prime}}, \omega_{3}\right) \mathrm{F}_{\mathrm{i}}(\omega) \mathrm{F}_{\mathrm{j}}(\omega) \mathrm{F}_{\mathrm{k}}(\omega) \mathrm{F}_{1}(\omega)+\ldots\right)$

Donde $\mu_{\mathrm{g}}$ es el momento dipolar del estado fundamental, $\alpha_{\mathrm{ij}}(\omega ; \omega)$ son las componentes del tensor polarizabilidad, siendo $\beta_{\mathrm{ijk}}\left(-\omega ; \omega_{1}, \omega_{2}\right)$ y $\gamma_{\mathrm{ijkl}}\left(-\omega ; \omega_{1}, \omega_{2^{\prime}} \omega_{3}\right.$ las componentes de los tensores primera y segunda hiperpolarizabilidad respectivamente. En estas ecuaciones se ha tenido en cuenta el convenio de Einstein que implica el sumatorio sobre todos los subíndices repetidos. A partir de la definición se puede ver que las propiedades moleculares $(\alpha$ y $\beta$ ) son una medida de la redistribución de carga cuando se le aplica un campo eléctrico al sistema. Por tanto, dependiendo de la simetría y del tipo de sustituyentes de la molécula, es lógico pensar que no todas las componentes de los tensores $\alpha$ y $\beta$ serán relevantes, sino que muchas componentes son nulas o se podrán despreciar. Así para la $\beta$ y en el caso de los donador-aceptor clásicos tenemos que, tomando como eje de referencia (eje z) la dirección del momento dipolar, la única componente no despreciable es $\beta_{z z z}$. En los cromóforos que poseen simetría $\mathrm{C} 2 v$ el momento dipolar tendrá la dirección del eje $\mathrm{C} 2$ de simetría (eje $\mathrm{z}$ ), y en este caso tendremos tres componentes no despreciables $\beta_{z y y}, \beta_{z y y}, \beta_{y z y}$

\section{PROCEDIMIENTO DE CÁLCULO}

Para la realización de los cálculos se ha utilizado el programa gaussian 98 [12]. En primer lugar se ha optimizado la geometría de todas las moléculas con el método Hartree-Fock restringido (RHF)[1] y el conjunto base 6-31G, seguido de un cálculo de las frecuencias vibracionales para asegurar que la geometría alcanzada es un mínimo real de energía. Las componentes del tensor $\beta$ se han calculado con tres métodos, el primero de ellos es el RHF, el método $a b$ initio más sencillo, cuyo defecto es que no incluye un adecuado tratamiento de la correlación electrónica. Este tratamiento se incluye en los otros dos métodos utilizados, la teoría de perturbaciones de Möller-Plesset de segundo orden (MP2)[1], que incluye un término de correción a la función de onda del RHF, y el funcional de la densidad SVWN [14], que incluye el funcional de intercambio de Slater y el funcional de correlación de Vosko-Will-Nusair. En todos los casos se ha empleado el conjunto base 6-31G.

A partir de la ecuación 2 es fácil deducir que las componentes del tensor $\beta$ se pueden obtener como derivadas de la energía respecto al campo eléctrico aplicado:

$$
\beta_{i \mathrm{ijk}}\left(-\omega ; \omega_{1}, \omega_{2}\right)=\varepsilon_{\mathrm{o}}^{-1}\left(\partial^{3} \mathrm{E} / \partial \mathrm{F}_{\mathrm{i}} \partial \mathrm{F}_{\mathrm{j}} \partial \mathrm{F}_{\mathrm{k}}\right)_{\mathrm{F}=0}
$$

Estas derivadas se pueden calcular tanto analítica como numéricamente. Ambos métodos consideran al campo eléctrico como una perturbación que se introduce en el hamiltoniano del sistema, habitualmente en el independiente del tiempo, por lo que los valores calculados son estáticos, sin dependencia de la frecuencia. En nuestro caso la derivada analítica se ha empleado en todos los cálculos con el
RHF, cuya expresión derivada de la teoría de perturbaciones es [8]

$$
\beta=<\Psi^{(0)}\left|\Sigma_{\mathrm{i}} \mathrm{r}_{\mathrm{i}}\right| \Psi^{(2)}>
$$

Donde $\Psi^{(0)} y \Psi^{(2)}$ son las funciones de onda del estado fundamental y perturbada de segundo orden respectivamente, y ri es la coordenada espacial del electrón $i$-ésimo. En el caso de los cálculos con los métodos MP2 y SVWN se ha empleado la técnica de campo finito $[8,1,15]$, ampliamente utilizada para el cálculo numérico de la derivada de la energía. Esta técnica necesita el cálculo de la energía del sistema para distintos valores de campo aplicado y posteriormente utiliza la expresión de la derivada numérica (ecuación 5), siendo importante tanto el valor de campo aplicado (F) como la precisión en el cálculo de la energía para evitar errores numéricos. En este trabajo se ha utilizado como campo $\mathrm{F}=0.001$ unidades atómicas y una precisión en la energía de 10.

$$
\beta=\left(E(2 F)-E(-2 F)-2(E(F)-E(-F))\left(2 F^{3}\right)^{-1}\right.
$$

Tanto los resultados del método analítico como el numérico proporcionan los valores de las componentes estáticas, para estimar los valores dinámicos se suele utilizar el modelo de los dos niveles [16]. Este modelo permite expresar la dependencia de las componentes con frecuencia como un producto de la componente estática por una función de dispersión que depende de la frecuencia de resonancia de la molécula $(\omega e g)$ y la frecuencia del campo electromagnético $(\omega)$ incidente sobre el material. Esta función es distinta para cada componente y efecto no lineal analizado. Para el caso particular del efecto electroóptico (analizado en este trabajo) las componentes de relevancia se pueden expresar como

$$
\begin{gathered}
\beta_{\mathrm{zzz}}(-\omega ; 0, \omega)=\beta_{\mathrm{zzz}}{ }^{0} \omega_{\mathrm{eg}}{ }^{2}\left(3 \omega_{\mathrm{eg}}{ }^{2}-\omega^{2}\right)\left(\omega_{\mathrm{eg}}{ }^{2}-\omega^{2}\right)^{-2} / 3 \\
\beta_{\mathrm{yzy}}(-\omega ; 0, \omega)=\beta_{\mathrm{yzy}}{ }^{0} \omega_{\mathrm{eg}}{ }^{2}\left(\omega_{\mathrm{eg}}{ }^{2}+\omega^{2}\right)\left(\omega_{\mathrm{eg}}{ }^{2}-\omega^{2}\right)^{-2} \\
\beta_{\mathrm{zyy}}(-\omega ; 0, \omega)=\beta_{\mathrm{yyz}}(-\omega ; 0, \omega)=\beta_{\mathrm{zyy}}{ }^{0} \omega_{\mathrm{eg}}{ }^{2}\left(\omega_{\mathrm{eg}}{ }^{2}-\omega^{2}\right)^{-1}
\end{gathered}
$$

Donde el superíndice 0 indica que se trata de la componente estática.

\section{RESULTADOS Y DISCUSIÓN}

Los valores de las componentes estáticas obtenidos con los distintos métodos se muestran en la tabla 1. Como ya se ha mencionado previamente, la simetría molecular hace que ciertas componentes sean nulas o despreciables, así para los cromóforos COU7, DMNPAA, DR1 y PNA únicamente se considera la componente $\beta_{z z z}{ }^{0}$. Los resultados de los tres métodos coinciden en el orden relativo, obteniéndose el mayor valor para el DR1, seguido del DMNPAA, COU7 y PNA respectivamente. En cuanto a los diferentes resultados entre métodos se puede señalar que el RHF (que no incluye el tratamiento de la correlación electrónica) es el que predice los valores inferiores. La inclusión de la correlación electrónica tanto en el MP2 como el SVWN produce un aumento en los valores obtenidos. Este aumento es especialmente significativo con el método SVWN para los compuestos DR1 y DMNPAA, que se atribuye a una sobreestimación de los métodos DFT cuando tenemos un sistema de tamaño grande con grupos donadores y aceptores de electrones [15]. En lo que respecta a la fiabilidad de los cálculos se puede decir que existe buena correlación entre los resultados obtenidos en este trabajo y los datos experimentales existentes en la bibliografía $\left(\beta_{z z z}{ }^{0}=4210 \mathrm{~m} / \mathrm{V}\right.$ para el DR1 [17] y $\beta_{\text {zzz }}{ }^{0}=2810 \mathrm{~m} / \mathrm{V}$ para el DMNPAA [18]), sobre todo en el caso del método MP2 / 6-31G, cuyos errores se pueden atribuir a las aproximaciones realizadas al realizar el cálculo para una molécula aislada, sin interacción con el entorno. Las 
otras dos moléculas estudiadas, $\mathrm{BM}$ y TIO, tienen como componentes no despreciables $\beta_{\text {yzy }}{ }^{0}, \beta_{\text {yyz }}{ }^{0}$ y $\beta_{\text {zyy }}$. En este caso, al contrario que en los anteriores cromóforos, los valores obtenidos con el RHF son superiores a los obtenidos con los métodos MP2 y SVWN. La relación entre moléculas en este caso tiene un resultado interesante, puesto que el cambio de los grupos $\mathrm{NH} 2$ de la tionina por los grupos $\mathrm{N}(\mathrm{CH} 3) 2$ del azul de metileno produce un aumento de un factor aproximado de 1.8 de los valores estimados.

TABLA 1: VALORES DE $B_{Z Z Z}{ }^{0}$ (PNA, DR1, DMNPAA Y COU7) Y B $_{Y Z Y}{ }^{0}(B M$ Y TIO) OBTENIDOS CON LOS DISTINTOS MÉTODOS DE CÁLCULO. UNIDADES $10 \mathrm{M} / \mathrm{V}$.

\begin{tabular}{|c|c|c|c|}
\hline Molécula & RHF/6-31G & MP2/6-31G & SVWN/6-31G \\
\hline BM & 10.4297 & 6.7267 & 4.2894 \\
\hline TIO & 6.4504 & 3.6782 & 2.3601 \\
\hline COU7 & 9.4784 & 23.6763 & 10.4234 \\
\hline DMNPAA & 15.5665 & 25.7427 & 67.3332 \\
\hline DR1 & 29.6803 & 55.9687 & 122.585 \\
\hline PNA & 4.5782 & 7.0126 & 5.9762 \\
\hline
\end{tabular}

En la figura 2 se representa la simulación de los coeficientes electroópticos microscópicos en función de la longitud de onda del campo electromagnético aplicado, para ello se han utilizado los valores del método MP2 / 6-31G y el modelo de los dos niveles (ecuaciones 6 y 7). Las longitudes de onda de resonancia empleadas en el modelo son las experimentales, $670 \mathrm{~nm}$ el BM, $610 \mathrm{~nm}$ la TIO, $433 \mathrm{~nm}$ la COU7, $400 \mathrm{~nm}$ el DMNPAA, $470 \mathrm{~nm}$ el DR1 y $371 \mathrm{~nm}$ la PNA.

Los resultados muestran que aún cuando el DR1 es el que posee valores superiores, los colorantes estudiados son potencialmente aplicables como cromóforos no lineales, así el BM presenta valores incluso superiores al DR1 para longitudes de onda del infrarrojo cercano, mientras que la COU7 tiene valores superiores al DMNPAA en longitudes de onda del verde. La tionina también posee valores superiores al DMNPAA en la zona del IR cercano, siendo los valores estimados para todos los colorantes superiores a los de la PNA, que es la molécula de referencia en óptica no lineal.

\section{CONCLUSIONES}

Se ha estudiado a nivel teórico la posible aplicación de tres colorantes clásicos (azul de metileno, tionina y coumarina 7) en dispositivos de óptica no lineal, comparándolos con tres cromóforos (disperse red 1, DMNPAA y p-nitroanilina) ampliamente empleados en dicho campo. En primer lugar se han calculado con los métodos RHF, MP2 y SVWN los valores de las principales componentes estáticas de los compuestos seleccionados, encontrando que para los colorantes los valores obtenidos son iguales o superiores a la molécula de referencia en óptica no lineal (PNA).

Posteriormente se ha empleado el modelo de los dos niveles a partir de los resultados obtenidos mediante el método MP2, simulando el comportamiento de las componentes del tensor $\beta$ para el efecto electroóptico a distintas frecuencias. Los resultados de la simulación en los colorantes estiman valores superiores a las moléculas no lineales en amplias zonas del espectro, por lo que puede estudiarse su aplicación en dispositivos fotónicos o materiales fotorrefractivos.

\section{AGRADECIMIENTOS}

Este trabajo ha sido financiado por los proyectos MAT2000-1361C04-03 y MAT99-0622 del Ministerio de Ciencia y Tecnología.

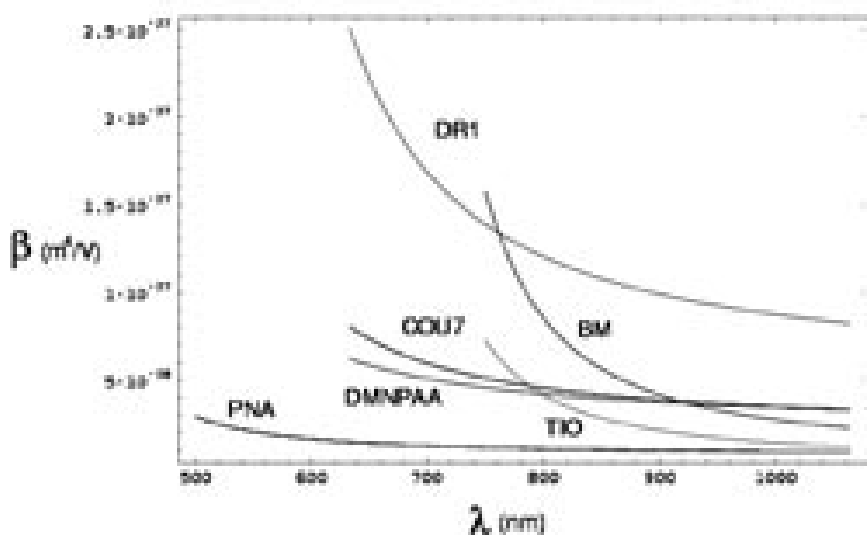

Figura 2: Representación de las componentes $\beta_{z z z}(-\omega ; 0, \omega)$ (para PNA, DR1, DMNPAA, COU7) y $\beta_{y z y}(-\omega ; 0, \omega)$ (para BM y TIO) en función de la longitud de onda. Se han utilizado los resultados del método MP2 / 6-31G y se ha aplicado el modelo de los dos niveles.

\section{BIBLIOGRAFÍA}

1. H. S. Nalwa, Handbook of advanced electronic and photonic materials and devices, Academic Press, New York, 2001.

2. P. Günter and J.-P. Huignard, "Photorefractive materials and their applications II," in Topics in applied physics, P. G\&\&ter and J.-P. Huignard, ed., Springer-Verlag, Berlin, 1989.

3. P. Günter, Nonlinear Optical Effects and Materials, Springer-Verlag, Berlin, 2000.

4. G. A. Lindsay y K. D. Singer, Polymers for second order nonlinear Optics, ACS Symposium series 601, 1995.

5. K. D. Singer, M. G. Kuzyk y J. E. Sohn, “Second-order nonlinear-optical processes in orientationally ordered materials: relationship between molecular and macroscopic properties," J. Opt. Soc. Am. B 4(6), pp. 968-976, 1987.

6. S. R. Marder, B. Kippelen, A. K.-Y. Jen y N. Peyghambarian, "Design and synthesis of chromophores and polymers for electro-optic and photorefractive applications," Nature 388, pp. 845-851, 1997.

7. D. M. Burland, R. D. Miller, O. Reiser, R. J. Twieg y C. A. Walsh, "The design, synthesis, and evaluation of chromophores for second-harmonic generation in a polymer waveguide," J. Appl. Phys. 71(1), pp. 410-417, 1992.

8. S. P. Karna y A. T. Yeates, eds., Nonlinear Optical Materials. Theory and Modeling, American Chemical Society, Washington, 1996.

9. M. Nakano, I. Shigemoto, S. Yamada y K. Yamaguchi, “Size-consistent approach and density analysis of hyperpolarizability-2nd hyperpolarizabilities of polymeric systems with and without deffects," J. Chem. Phys. 10, pp. 4175-4191, 1995.

10. P. Acebal, S. Blaya y L. Carretero, "Theoretical study of second-order non-linear optical propeties of pyrromethene dyes for photonic application," J. Phys. B: At. Mol. Opt. Phys. 36, pp. 2445-2454, 2003.

11. P. Acebal, S. Blaya y L. Carretero, "Ab initio study of absorption and emission spectra of pm567," Chem. Phys. Lett. 374, pp. 206-214, 2003.

12. M. J. Frisch, G. W. Trucks, H. B. Schlegel, G. E. Scuseria, M. A. Robb, J. R. Cheeseman, V. G. Zakrzewski, J. A. Montgomery, R. E. Stratmann, J. C. Burant, S. Dapprich, J. M. Millam, A. D. Daniels, K. N. Kudin, M. C. Strain, O. Farkas, J. Tomasi, V. Barone, M. Cossi, R. Cammi, B. Menucci, C. Pomelli, C. Adamo, S. Clifford, J. Ochterski, G. A. Petereson, P. Y. Ayala, Q. Cui, K. Morokuma, D. K. Malick, A. D. Rabuck, K. Raghavachari, J. B. Foresman, J. Cioslowski, J. V. Ortiz, B. B. Stefanov, G. Liu, A. Liashenko, P. Piskorz, I. Komaromi, R. Gomperts, R. L. Martin, D. J. Fox, T. Keith, M. A. Al-Laham, C. Y. Peng, A. Nanayakkara, C. Gonzalez, M. Challacombe, P. M. W. Gill, B. G. Johnson, W. Chen, M. W. Wong, J. L. Andres, M. HeadGordon, E. S. Replogle y J. A. Pople. GAUSSIAN 98, Revision A.7, Gaussian, Inc, Pittsburg PA, 1998.

13. W. J. Hehre, L. Radom, P. V. R. Schleyer y J. A. Pople, Ab initio molecular orbital theory, John Wiley \& Sons Inc., New York, 1986.

14. E. Baerends y O. Gritsenko, "A quantum chemical view of density functional theory," J. Phys. Chem. A 101(30), pp. 5383-5403, 1997.

15. B. Champagne, E. Perpete, D. Jacquemin, S. J. A. V. Gisbergen, E. Baerends, C. Soubra-Ghaoui, K. A. Robins y B. Kirtman, "Assesment of conventional density functional schemes for computing the dipole moment and (hyper)polarizabilities of push-pull $\pi$-conjugated systems," J. Phys. Chem A 104, pp. 4755-4763, 2000.

16. J. L. Oudar y D. S. Chemla, "Hyperpolarizabilities of the nitroanilines and their relations to the excited state dipole moment," J. Chem. Phys. 66(6), pp. 2664-2668, 1977.

17. C. R. Moylan, R. J. Twieg, V. Y. Lee, S. A. Swanson, K. M. Betterton y R. D. Miller, "Nonlinear optical chromophores with large hyperpolarizabilities and enhanced thermal stabilities," J. Am. Chem. Soc. 115, pp. 12599-12600, 1993.

18. C. R. Moylan, R. Wortmann, R. J. Twieg y I.-H. Comb, "Improved characterization of chromophores for photorefractive aplications," J. Opt. Soc. Am. B 15(2), pp. 929-932, 1998. 